PROCEEDINGS OF THE

AMERICAN MATHEMATICAL SOCIETY

Volume 136, Number 8, August 2008, Pages 2655-2663

S 0002-9939(08)09408-2

Article electronically published on April 2, 2008

\title{
A NOTE ON SERRE'S THEOREM IN GROUP COHOMOLOGY
}

\author{
ERGÜN YALÇIN
}

(Communicated by Jonathan I. Hall)

\begin{abstract}
In 1987, Serre proved that if $G$ is a $p$-group which is not elementary abelian, then a product of Bocksteins of one dimensional classes is zero in the mod $p$ cohomology algebra of $G$, provided that the product includes at least one nontrivial class from each line in $H^{1}\left(G, \mathbb{F}_{p}\right)$. For $p=2$, this gives that $\left(\sigma_{G}\right)^{2}=0$, where $\sigma_{G}$ is the product of all nontrivial one dimensional classes in $H^{1}\left(G, \mathbb{F}_{2}\right)$. In this note, we prove that if $G$ is a nonabelian 2-group, then $\sigma_{G}$ is also zero.
\end{abstract}

\section{INTRODUCTION}

Let $G$ be a finite $p$-group and let $H^{*}\left(G, \mathbb{F}_{p}\right)$ denote the cohomology algebra of $G$ in $\bmod p$ coefficients. One of the most important theorems about the algebra structure of $H^{*}\left(G, \mathbb{F}_{p}\right)$ is Serre's theorem [6] which states that if $G$ is not an elementary abelian group, then there exist nontrivial one dimensional classes $x_{1}, x_{2}, \ldots, x_{m} \in H^{1}\left(G, \mathbb{F}_{p}\right)$ such that $\beta\left(x_{1}\right) \beta\left(x_{2}\right) \cdots \beta\left(x_{m}\right)=0$. The original proof by Serre is an existence proof giving no indication of how such a vanishing product should be chosen. In his second proof, Serre gives an explicit set of one dimensional classes which has a vanishing product. He proves the following:

Theorem 1.1 (Serre 7). Let $G$ be a p-group which is not elementary abelian. Then the product $\prod \beta(x)$ is zero if the product includes at least one nontrivial class from each line in $H^{1}\left(G, \mathbb{F}_{p}\right)$.

When $G$ is a 2-group, we have $\beta(x)=x^{2}$ for each $x \in H^{1}\left(G, \mathbb{F}_{2}\right)$, so Theorem 1.1 gives $\left(\sigma_{G}\right)^{2}=0$, where

$$
\sigma_{G}=\prod_{x \in H^{1}\left(G, \mathbb{F}_{2}\right)-\{0\}} x .
$$

The class $\sigma_{G}$ itself is zero for many 2-groups, but there are also examples of 2-groups where $\sigma_{G}$ is not zero. For example, if $G \cong \mathbb{Z} / 4 \times \mathbb{Z} / 2$, then $\sigma_{G}$ is not zero. In this note, we show that such examples are limited to abelian 2-groups. Our main result is the following:

Theorem 1.2. If $G$ is a nonabelian 2-group, then $\sigma_{G}=0$.

Received by the editors March 12, 2007.

2000 Mathematics Subject Classification. Primary 20J06.

Key words and phrases. Cohomology of groups, Stiefel-Whitney classes, essential cohomology. The author was partially supported by TÜBİTAK-BDP and TÜBA-GEBİ/2005-16. 
This theorem has many applications in group cohomology. Our main motivation for studying this version of Serre's theorem is a calculation related to the StiefelWhitney classes of the regular representation of a 2-group. As a consequence of Theorem 1.2, we obtain that the top Stiefel-Whitney class of the augmentation module for the regular representation of a nonabelian 2-group is zero. We also give some other applications related to essential cohomology. All these applications are explained in Section 4 .

The proof of Theorem 1.2 is very similar to the proof of Theorem 1.1, although there are some subtle differences. In the proof of Theorem 1.1, Serre uses the fact that if $G$ is a $p$-group which is not elementary abelian, then there exist some two dimensional relations of the form

$$
\sum_{k} a_{k} \beta\left(x_{k}\right)+\sum_{i<j} c_{i j} x_{i} x_{j}=0
$$

where $\left\{x_{1}, \ldots, x_{n}\right\}$ is a basis for $H^{1}\left(G, \mathbb{F}_{p}\right)$. But the existence of such relations are no longer enough to prove Theorem 1.2 Some complications arise when $G$ is a nonabelian 2-group which has no two dimensional relations with some of the $c_{i j}$ 's nonzero. For example, the modular group

$$
M_{2^{n}}=\left\langle a, b \mid a^{2}=b^{2^{n-1}}=1, a b a=b^{2^{n-2}+1}\right\rangle
$$

of order $2^{n}$ with $n \geq 4$ is such a group (see Remark 3.3). To overcome this problem, we prove the following:

Proposition 1.3. Let $G$ be a 2-group and let $\left\{x_{1}, \ldots, x_{n}\right\}$ be a basis for $H^{1}\left(G, \mathbb{F}_{2}\right)$. If $G$ is nonabelian, then there exists a nontrivial relation of the form

$$
\sum_{i<j} c_{i j}\left(x_{i}^{2} x_{j}+x_{j}^{2} x_{i}\right)=0
$$

in $H^{3}\left(G, \mathbb{F}_{2}\right)$.

To prove Proposition 1.3, we consider group cohomology in integer coefficients and show that the inflation map

$$
\text { inf : } H^{3}(G / \Phi(G), \mathbb{Z}) \rightarrow H^{3}(G, \mathbb{Z})
$$

has a nontrivial kernel when $G$ is a nonabelian p-group (here $\Phi(G)$ denotes the Frattini subgroup of $G$ ). We can also state this in the following way:

Proposition 1.4. Let $G$ be a p-group. Then the following are equivalent:

(i) $G$ is abelian.

(ii) The map inf : $H^{2}(G / \Phi(G), \mathbb{Q} / \mathbb{Z}) \rightarrow H^{2}(G, \mathbb{Q} / \mathbb{Z})$ is injective.

This is similar to a criterion given by Serre in [6] for a $p$-group to be elementary abelian (see Proposition 3.4).

This paper is organized as follows: In Section 2, we go through Serre's arguments to show how Theorem 1.2 follows from Proposition 1.3. Propositions 1.3 and 1.4 are proved in Section 3. In Section 4, we describe some consequences of Theorem 1.2.

\section{Proof of the MAIN THEOREM}

Let $G$ be a 2 -group and let $\left\{x_{1}, \ldots, x_{n}\right\}$ be a basis for $H^{1}\left(G, \mathbb{F}_{p}\right)$. We can express $\sigma_{G}$ as the product

$$
\sigma_{G}=\prod_{\left(a_{1}, \ldots, a_{n}\right) \in\left(\mathbb{F}_{2}\right)^{n}-\{0\}}\left(a_{1} x_{1}+\cdots+a_{n} x_{n}\right) .
$$


Note that $\sigma_{G}$ can also be expressed as the determinant of the matrix $A$ defined by

$$
A_{i j}=x_{j}^{2^{i-1}} \text { for } 1 \leq i, j \leq n .
$$

This is because both $\sigma_{G}$ and $\operatorname{det}(A)$ have the same degree and the same zero set as polynomials in $x_{1}, \ldots, x_{n}$ over the algebraic closure of $\mathbb{F}_{2}$. Note that both polynomials are zero at a point $\alpha \in\left(\overline{\mathbb{F}}_{2}\right)^{n}$ if and only if the coordinates of $\alpha$ satisfy a linear equation of the form $a_{1} x_{1}+\cdots+a_{n} x_{n}=0$ for some $\left(a_{1}, \ldots, a_{n}\right) \in\left(\mathbb{F}_{2}\right)^{n}-\{0\}$.

Let $M$ denote the $n$-dimensional free module over the cohomology algebra $H^{*}\left(G, \mathbb{F}_{2}\right)$. For $i=1, \ldots, n$, let $e_{i}$ denote the vector

$$
e_{i}=\left(x_{i}, x_{i}^{2}, \ldots, x_{i}^{2^{n-1}}\right)
$$

in $M$. Since the column vector given by $e_{i}$ is the $i$-th column of $A$, we have

$$
\operatorname{det}(A)=e_{1} \wedge e_{2} \wedge \cdots \wedge e_{n}
$$

in the $n$-th exterior power $\bigwedge^{n} M \cong H^{*}\left(G, \mathbb{F}_{p}\right)$. Suppose there is a nontrivial relation

$$
\sum_{i<j} c_{i j}\left(e_{i} \wedge e_{j}\right)=0
$$

in $M \wedge M$, say with $c_{12} \neq 0$. Then by taking exterior product with $e_{3} \wedge \cdots \wedge e_{n}$, we get $\operatorname{det}(A)=0$. So, to complete the proof of Theorem 1.2, we need to show that there is a nontrivial relation in $M \wedge M$.

Lemma 2.1 (cf. Serre [7). Let $G$ be a 2-group. If there is a nontrivial relation of the form

$$
\sum_{i<j} c_{i j}\left(x_{i}^{2} x_{j}+x_{i} x_{j}^{2}\right)=0
$$

in $H^{3}\left(G, \mathbb{F}_{2}\right)$, then there also exists a nontrivial relation in $M \wedge M$.

Proof. Let $c_{i j}$ be the coefficients of a nontrivial relation in $H^{3}\left(G, \mathbb{F}_{2}\right)$. Define

$$
L_{k l}=\sum_{i<j} c_{i j}\left(x_{i}^{2^{k}} x_{j}^{2^{l}}+x_{i}^{2^{l}} x_{j}^{2^{k}}\right)
$$

for $k, l \geq 0$. Note that there is a relation in $M \wedge M$ of the form given in equation (3) if and only if $L_{k l}=0$ for all $k, l \geq 0$. The class $L_{00}$ is zero by trivial reasons and $L_{10}$ is zero by assumption. For $m \geq 2$, we have

$$
L_{m 0}=S q^{2^{m-1}} \cdots S q^{4} S q^{2}\left(L_{10}\right) .
$$

So, $L_{m 0}=0$ for all $m \geq 0$. Now, suppose $k$ and $l$ are such that $k=l+m$ for some $m \geq 0$. Then, $L_{k l}=\left(L_{m 0}\right)^{2^{l}}=0$. Since $L_{k l}=L_{l k}$, we conclude that $L_{k l}=0$ for all $k, l \geq 0$.

This shows that the proof of Theorem 1.2 is complete once we prove Proposition 1.3 . 


\section{Proof of Proposition 1.3}

As before, we assume that $G$ is a 2 -group. Let $\left\{x_{1}, \ldots, x_{n}\right\}$ be a basis for $H^{1}\left(G, \mathbb{F}_{p}\right)$. Recall that the inflation map

$$
\inf : H^{*}\left(G / \Phi(G), \mathbb{F}_{2}\right) \rightarrow H^{*}\left(G, \mathbb{F}_{2}\right)
$$

is bijective in dimension one. For each $x_{i} \in H^{1}\left(G, \mathbb{F}_{2}\right)$, we denote the associated class in $H^{1}\left(G / \Phi(G), \mathbb{F}_{2}\right)$ also by $x_{i}$. Note that to prove Proposition 1.3 , it is enough to show that there is an element of the form

$$
\sum_{i<j} c_{i j}\left(x_{i}^{2} x_{j}+x_{i} x_{j}^{2}\right)
$$

in $H^{3}\left(G / \Phi(G), \mathbb{F}_{2}\right)$ which lies in the kernel of the inflation map. We show this using integral cohomology. The quotient group $G / \Phi(G)$ is an elementary abelian 2-group, and the integral cohomology rings of elementary abelian 2-groups are well understood. In particular, it is known that $H^{3}(G / \Phi(G), \mathbb{Z})$ is generated by $\left\{u_{i j} \mid 1 \leq i<j \leq n\right\}$ where the mod 2-reduction of $u_{i j}$ is equal to $x_{i}^{2} x_{j}+x_{i} x_{j}^{2}$ in $H^{3}\left(G, \mathbb{F}_{2}\right)$ (see, for example, [2]). Consider the following diagram:

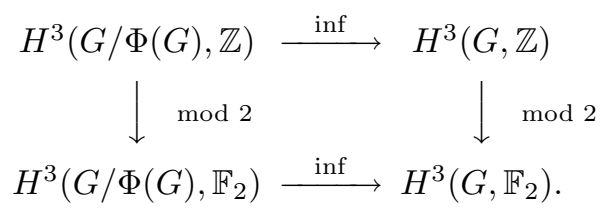

If the kernel of the inflation map in integer coefficients is nonzero, then there is a nontrivial class $\sum_{i<j} c_{i j} u_{i j}$ in $H^{3}(G / \Phi(G), \mathbb{Z})$ whose mod 2 reduction $\sum_{i<j} c_{i j}\left(x_{i}^{2} x_{j}+x_{i} x_{j}^{2}\right)$ lies in the kernel of the mod 2 inflation map. Hence the proof of Proposition 1.3 is complete once we prove the following:

Proposition 3.1. If $G$ is a nonabelian p-group, then the kernel of the inflation map

$$
\inf : H^{3}(G / \Phi(G), \mathbb{Z}) \rightarrow H^{3}(G, \mathbb{Z})
$$

is nontrivial.

Proof. The statement is equivalent to the statement that the kernel of the inflation map

$$
\inf : H^{2}(G / \Phi(G), \mathbb{Q} / \mathbb{Z}) \rightarrow H^{2}(G, \mathbb{Q} / \mathbb{Z})
$$

is nontrivial. Consider the 5 -term exact sequence

$$
0 \rightarrow H^{1}(G / \Phi(G)) \stackrel{\text { inf }}{\longrightarrow} H^{1}(G) \stackrel{\text { res }}{\longrightarrow} H^{1}(\Phi(G))^{G} \stackrel{d_{2}}{\longrightarrow} H^{2}(G / \Phi(G)) \stackrel{\text { inf }}{\longrightarrow} H^{2}(G)
$$

where all the cohomology groups are with coefficients in $\mathbb{Q} / \mathbb{Z}$. Note that

$$
H^{1}(G, \mathbb{Q} / \mathbb{Z})=\operatorname{Hom}(G, \mathbb{Q} / \mathbb{Z})=\operatorname{Hom}(G /[G, G], \mathbb{Q} / \mathbb{Z})=(G /[G, G])^{*},
$$

where $(G /[G, G]) *$ denotes the dual group of the abelian group $G /[G, G]$. The above 5 -term exact sequence gives an exact sequence of the form

$$
0 \rightarrow(G / \Phi(G))^{*} \stackrel{\inf }{\longrightarrow}(G /[G, G])^{*} \longrightarrow\left((\Phi(G) /[\Phi(G), \Phi(G)])^{*}\right)^{G} \longrightarrow \operatorname{ker}(\inf ) \rightarrow 0 .
$$


From this it is easy to see that the cokernel of the first inflation map is $(\Phi(G) /[G, G])^{*}$. Also note that

$$
\begin{aligned}
\left((\Phi(G) /[\Phi(G), \Phi(G)])^{*}\right)^{G} & =(\operatorname{Hom}(\Phi(G), \mathbb{Q} / \mathbb{Z}))^{G}=\operatorname{Hom}_{G}(\Phi(G), \mathbb{Q} / \mathbb{Z}) \\
& =\operatorname{Hom}(\Phi(G) /[\Phi(G), G], \mathbb{Q} / \mathbb{Z})=(\Phi(G) /[\Phi(G), G])^{*} .
\end{aligned}
$$

Thus, the kernel of inflation map in degree 2 is isomorphic to the group

$$
([G, G] /[\Phi(G), G])^{*} \text {. }
$$

Now, the result follows from the following group theoretical lemma.

Lemma 3.2. If $G$ is a nonabelian p-group, then $[G, G] \neq[\Phi(G), G]$.

Proof. Let $G$ be a minimal counterexample to this lemma. Then $G$ is a nonabelian $p$-group with $[G, G]=[\Phi(G), G]$. Let $H$ be a normal subgroup of $G$ such that $H \leq[G, G]$ with index $p$. Note that $G / H$ is a nonabelian $p$-group satisfying

$$
[G / H, G / H]=[G, G] / H=[\Phi(G), G] / H=[\Phi(G) / H, G / H]=[\Phi(G / H), G / H] .
$$

So, by the minimality of $G$, we must have $H=1$. This gives that $[G, G]=[\Phi(G), G]$ is a cyclic group of order $p$. In a $p$-group, a normal subgroup of order $p$ is always central, so we have $[G, G] \leq Z(G)$. Under these conditions, $\left[a^{p}, b\right]=[a, b]^{p}=1$ for every $a, b \in G$. Thus, the subgroup $G^{(p)}$ generated by $p$-th powers is also a central subgroup of $G$. Note that $\Phi(G)=[G, G] G^{(p)}$, so we obtain that $\Phi(G) \leq$ $Z(G)$, which is in contradiction with our assumption since $[\Phi(G), G]=[G, G]$ is nontrivial.

This completes the proof of Proposition 1.3 and hence the proof of Theorem 1.2 .

Remark 3.3. In the proof of Theorem 1.1 given in [7, Steenrod operations are applied to a relation of the form

$$
\sum_{k} a_{k} \beta\left(x_{k}\right)+\sum_{i<j} c_{i j} x_{i} x_{j}=0
$$

to produce higher dimensional relations which eventually forces $\prod \beta(x)$ to be zero. But, the argument with Steenrod operations works only when some of the $c_{i j}$ 's are nonzero. Because of this, the case where all $c_{i j}$ 's are zero is considered separately. In that case, one has $\sum_{k} a_{k} \beta\left(x_{k}\right)=0$ which gives $\beta\left(\sum_{k} a_{k} x_{k}\right)=0$. So, Theorem 1.1 still holds if $G$ has no relations with some $c_{i j}$ 's nonzero. However, this causes a problem with the proof of Theorem 1.2. One may expect that if $G$ is nonabelian, then there should exist at least one relation of the above form with some $c_{i j}$ 's nonzero, but unfortunately this is not true. The modular group

$$
M_{2^{n}}=\left\langle a, b \mid a^{2}=b^{2^{n-1}}=1, a b a=b^{2^{n-2}+1}\right\rangle
$$

of order $2^{n}$ with $n \geq 4$ has no relation of the above form with some $c_{i j}$ 's nonzero. The only two dimensional relation that $M_{2^{n}}$ has in its mod 2 cohomology is $\beta(u)=$ 0 , where $u \in H^{1}\left(M_{2^{n}}, \mathbb{F}_{2}\right)$ is the class defined by the homomorphism $u\left(a^{i} b^{j}\right)=$ $j \bmod 2$.

Note that if $G$ is abelian, then $[G, G]=[\Phi(G), G]=1$, and hence the kernel of the inflation map is trivial. So, the proof of Proposition 1.4 is also complete. Proposition 1.4 has formal similarities to the following result by Serre. 
Proposition 3.4 (Proposition 3 in [6]). Let $G$ be a p-group. Then, the following are equivalent:

(i) $G$ is elementary abelian.

(ii) The inflation map inf: $H^{2}\left(G / \Phi(G), \mathbb{F}_{p}\right) \rightarrow H^{2}\left(G, \mathbb{F}_{p}\right)$ is injective.

We can interpret Proposition 1.4 as a similar criterion for being abelian. Another way to interpret Proposition 3.1 is to say that the image of the differential $d_{2}$ is nontrivial in the Hochschield-Serre spectral sequence for the extension

$$
0 \rightarrow \Phi(G) \rightarrow G \rightarrow G / \Phi(G) \rightarrow 0
$$

of a nonabelian 2-group $G$. In this sense, Proposition 3.1 is a generalization of Proposition 7.2 in 9 .

Remark 3.5. There is an alternative approach to the proof Proposition 1.3 which was suggested to us by the anonymous referee. Since it gives more insight to this proposition, we briefly explain it here. Consider the quotient group $F=$ $G /[\Phi(G), G]$ which fits into a central extension of the form $1 \rightarrow \Phi(F) \rightarrow F \rightarrow E \rightarrow$ 1 where $E=G / \Phi(G)$. By Lemma 3.2, $F$ is nonabelian. Since $\Phi(F)$ is central, $F$ has a nonabelian quotient $Q$ which fits into a central extension of the form

$$
\mathcal{E}: 1 \rightarrow C \rightarrow Q \rightarrow E \rightarrow 1
$$

where $C$ is cyclic. By taking further quotients if necessary, we can assume that $Q / C_{2}$ is abelian where $C_{2}$ is the unique subgroup of $C$ of order 2. Let $\alpha \in H^{2}(E, C)$ be the extension class for $\mathcal{E}$. Consider the long exact sequence

$$
\cdots \rightarrow H^{2}(G, \mathbb{Z} / 2) \rightarrow H^{2}(E, \bar{C}) \rightarrow H^{2}(E, C) \stackrel{d}{\longrightarrow} H^{3}(E, \mathbb{Z} / 2) \rightarrow \cdots
$$

associated to the short exact sequence $0 \rightarrow \mathbb{Z} / 2 \rightarrow \bar{C} \rightarrow C \rightarrow 0$ where $\bar{C}$ is a cyclic group of order $2|C|$. We claim that $d(\alpha)$ is nonzero. Assume the contrary, that $d(\alpha)=0$. Then, the extension $\mathcal{E}$ will lift to a central extension of the form $1 \rightarrow \bar{C} \rightarrow \bar{Q} \rightarrow E \rightarrow 1$. Note that since the commutator subgroup of $\bar{Q}$ is central, we have $[a, b]^{2}=\left[a^{2}, b\right]=1$ for every $a, b \in \bar{Q}$. This gives that $Q=\bar{Q} /(\mathbb{Z} / 2)$ is abelian which contradicts our starting assumption. This proves $d(\alpha)$ is nonzero. It is clear that inflation of $d(\alpha)$ to $G$ is zero, because $\inf _{E}^{G}(\alpha)=0$. So, it remains to show that $d(\alpha)$ is in the desired form. For this it is enough to show that $d(\alpha)$ is in the image of the Bockstein operator. Since $Q / C_{2}$ is abelian, there is a class $\gamma \in H^{2}(E, C)$ such that $d(\gamma)=0$ and that $\pi_{*}(\gamma)=\pi_{*}(\alpha)$ under the homomorphism $\pi_{*}: H^{2}(E, C) \rightarrow H^{2}\left(E, C / C_{2}\right)$. So, $\alpha^{\prime}=\alpha-\gamma$ is in the image of $j_{*}: H^{2}\left(E, C_{2}\right) \rightarrow$ $H^{2}(E, C)$ and $d(\alpha)=d\left(\alpha^{\prime}\right)$. If $\alpha^{\prime}=j_{*}(u)$, then an easy diagram chase shows that $d\left(\alpha^{\prime}\right)=\beta(u)$. This completes the proof that $d(\alpha)$ is in the desired form, and hence completes the argument.

\section{Consequences of the main theorem}

The converse of Theorem 1.2 does not hold since there are some abelian groups with $\sigma_{G}=0$. For example, when $G=\mathbb{Z} / 4 \times \mathbb{Z} / 4$, we have

$$
H^{*}\left(G, \mathbb{F}_{2}\right) \cong \bigwedge\left(x_{1}, x_{2}\right) \otimes \mathbb{F}_{2}\left[y_{1}, y_{2}\right]
$$

so $\sigma_{G}=x_{1} x_{2}\left(x_{1}+x_{2}\right)=0$. In general, if $G$ is an abelian group with more than two factors with exponent greater than or equal to 4 , then $\sigma_{G}=0$. On the other hand, it is easy to see that $\sigma_{G} \neq 0$ when $G \cong \mathbb{Z} / 2^{k} \times(\mathbb{Z} / 2)^{n}$ for some $n \geq 0$ and $k \geq 1$. Thus, we conclude: 
Corollary 4.1. Let $G$ be a 2-group. Then $\sigma_{G} \neq 0$ if and only if $G \cong \mathbb{Z} / 2^{k} \times(\mathbb{Z} / 2)^{n}$ for some $n \geq 0$ and $k \geq 1$.

This gives a complete description of 2-groups with $\sigma_{G} \neq 0$. The element $\sigma_{G}$ is also defined for $p$-groups with $p>2$, but it is easy to see that $\sigma_{G}=0$ for all $p$-groups in this case.

Corollary 4.1 suggests another way to prove Theorem 1.2. Since abelian 2-groups with $\sigma_{G} \neq 0$ are in a special form, a minimal counterexample to Theorem 1.2 would be a nonabelian 2-group $G$ which fits into an extension of the form

$$
1 \rightarrow \mathbb{Z} / 2 \rightarrow G \rightarrow \mathbb{Z} / 2^{k} \times(\mathbb{Z} / 2)^{n} \rightarrow 1
$$

for some $n \geq 0$ and $k \geq 1$. One can argue directly that such groups have some nontrivial three dimensional relations of the form given in Proposition 1.3. The proof we give in Section 3 is more conceptual and explains why such three dimensional relations exist.

4.1. The top Stiefel-Whitney class of an augmented regular representation. Our main motivation for obtaining the vanishing product in Theorem 1.2 is a calculation related to the Stiefel-Whitney classes of regular representations. Let

$$
I_{G}=\operatorname{ker}\{\varepsilon: \mathbb{R} G \longrightarrow \mathbb{R}\}
$$

denote the augmentation ideal of the regular representation of $G$ over real numbers. We are interested in calculating the top Stiefel-Whitney class of $I_{G}$.

This calculation is important for two reasons. First, this calculation is related to a question asked by Reiner and Webb. In [5], Reiner and Webb define a cohomology class $\zeta_{G}$ as the extension class of the subset complex of $G$ and ask for which groups $\zeta_{G}$ is nonzero. We showed in [3] that the mod 2-reduction of $\zeta_{G}$ is the top-Stiefel Whitney class of $I_{G}$. So, calculation of the top Stiefel-Whitney class of $I_{G}$ has direct consequences for the class $\zeta_{G}$. Another reason for calculating the top StiefelWhitney class of $I_{G}$ is a theorem of Turygin which says that there are Borsuk-Ulam type theorems for group actions on products of spheres if the top Stiefel-Whitney class of $I_{G}$ pulls back to a nonzero class in the associated equivariant cohomology (see [8] for details).

Now, we explain how Theorem 1.2 applies to the calculation of Stiefel-Whitney classes. Let $w_{\text {top }}\left(I_{G}\right)$ denote the top Stiefel-Whitney class of $I_{G}$. Note that

$$
I_{G}=I_{G / \Phi(G)} \oplus \operatorname{ind}_{\Phi(G)}^{G}\left(I_{\Phi(G)}\right) .
$$

So, by the multiplicative property of the top Stiefel-Whitney class, we have

$$
w_{\text {top }}\left(I_{G}\right)=w_{\text {top }}\left(I_{G / \Phi(G)}\right) \cdot w_{\text {top }}\left(\operatorname{ind}_{\Phi(G)}^{G}\left(I_{\Phi(G)}\right)\right) .
$$

Recall that

$$
I_{G / \Phi(G)}=\bigoplus_{\xi \in \operatorname{Hom}\left(G, \mathbb{R}^{\times}\right)-\{0\}} \xi
$$

and the first Stiefel-Whitney class $w_{1}(\xi) \in H^{1}\left(G, \mathbb{F}_{2}\right)$ is the class associated to $\xi$ under the following isomorphisms:

$$
H^{1}\left(G, \mathbb{F}_{2}\right) \cong \operatorname{Hom}(G, \mathbb{Z} / 2) \cong \operatorname{Hom}\left(G, \mathbb{R}^{\times}\right) .
$$

So, we have

$$
w_{\text {top }}\left(I_{G / \Phi(G)}\right)=\prod_{\xi \in \operatorname{Hom}\left(G, \mathbb{R}^{\times}\right)-\{0\}} w_{1}(\xi)=\sigma_{G} .
$$


As a consequence of Theorem 1.2. we obtain the following:

Corollary 4.2. If $G$ is a nonabelian 2-group, then $w_{\text {top }}\left(I_{G}\right)=0$.

This was also proved in [3] using different methods.

4.2. Inflated essential classes. The essential cohomology ideal of a $p$-group $G$ is defined as the ideal

$$
\mathcal{E}_{s s^{*}}(G)=\operatorname{ker}\left\{\prod_{H<G} \operatorname{res}_{H}^{G}: H^{*}\left(G, \mathbb{F}_{p}\right) \rightarrow \prod_{H<G} H^{*}\left(H, \mathbb{F}_{p}\right)\right\} .
$$

Note that if $N$ is a normal subgroup of $G$ such that $N \leq \Phi(G)$, then

$$
\inf _{G / N}^{G}\left(\mathcal{E} s s^{*}(G / N)\right) \subseteq \mathcal{E} s s^{*}(G) .
$$

We would like to know if these inflated essential classes give some interesting essential classes in $\mathcal{E} s s^{*}(G)$. We consider the case where $G$ is 2-group and $N=\Phi(G)$. Note that

$$
H^{*}\left(G / \Phi(G), \mathbb{F}_{2}\right) \cong \mathbb{F}_{2}\left[x_{1}, \ldots, x_{k}\right]
$$

where $k=\operatorname{dim}\left(H^{1}\left(G, \mathbb{F}_{2}\right)\right)$. It is easy to see that the essential ideal of an elementary abelian 2-group is the principle ideal generated by the product of all nontrivial one dimensional classes. So, $\inf _{G / \Phi(G)}^{G}(u)$ is divisible by $\sigma_{G}$. Another corollary of Theorem 1.2 is the following:

Corollary 4.3. If $G$ is a nonabelian 2-group, then

$$
\inf _{G / \Phi(G)}^{G}\left(\mathcal{E} s s^{*}(G / \Phi(G))\right)=0 .
$$

This corollary has some further applications in terms of essential classes coming from relative cohomology classes. For a $G$-set $X$, there is an $X$-relative version of the usual group cohomology. The usual cohomology algebra of a $p$-group $G$ with coefficients in a field $k=\mathbb{F}_{p}$ is isomorphic to the algebra $\operatorname{Ext}_{k G}^{*}(k, k)$ of the equivalence classes of $k$ by $k$ extensions of $k G$-modules. If we require the extensions to be $X$-split (i.e., split after tensoring with a permutation module $k X$ ), then we get an $X$-relative version of the usual $\operatorname{Ext}_{k G}^{*}(k, k)$, which is usually denoted by $\operatorname{XExt}_{k G}^{*}(k, k)$. There is an obvious map

$$
\varphi: \operatorname{XExt}_{k G}^{*}(k, k) \rightarrow \operatorname{Ext}_{k G}^{*}(k, k)
$$

which takes an $X$-relative extension to a usual $k G$-module extension. It is shown in 1 that if $G$ is $p$-group and $X$ is the disjoint union of all $G$-sets of the form $G / H$

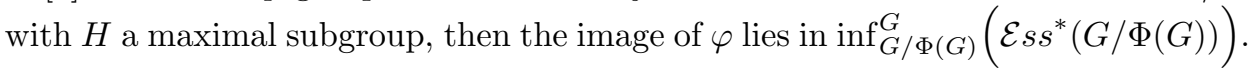
As a consequence of Corollary [4.3, it is obtained in [1] that $\varphi$ is a zero map when $G$ is a nonabelian 2-group.

4.3. Minh's theorem on essential cohomology. After proving Theorem 1.2 , we noticed that in [4 Minh uses the statement of Theorem 1.2 to prove the $p=2$ case of the following theorem:

Theorem 4.4 (Minh [4). Let $G$ be a p-group. If $u \in \mathcal{E} s s^{*}(G)$, then $u^{p}=0$.

Minh refers to Serre's theorem for the proof of Theorem 1.2, but, as we explained in Remark 3.3. the proof of Theorem 1.2 does not follow directly from Serre's arguments in [7. So, the $p=2$ case of the above theorem can be considered as another application of Theorem 1.2 , 


\section{ACKNOWLEDGEMENTS}

I would like to thank David Green and Peter Symonds for reading and commenting on earlier versions of this paper. I also thank the referee for a careful reading of the manuscript and for suggesting the alternative proof for Proposition 1.3 described in Remark 3.5

\section{REFERENCES}

[1] F. Altunbulak, Relative extension classes and essential cohomology, Ph.D. thesis, in preparation.

[2] D. Benson and J. F. Carlson, The cohomology of extraspecial groups, Bull. London Math. Soc. 24 (1992), 209-235. MR 1157256 (93b:20087)

[3] A. Güçlükan and E. Yalçın, The Euler class of a subset complex, preprint, 2007.

[4] P. A. Minh, Essential mod-p cohomology classes of p-groups: An upper bound for nilpotency degrees, Bull. London Math. Soc. 32 (2000), 285-291. MR.1750170 (2001c:20106)

[5] V. Reiner and P. Webb, The combinatorics of the bar resolution in group cohomology, J. Pure Appl. Algebra 190 (2004), 291-327. MR2043333 (2005i:57001)

[6] J. P. Serre, Sur la dimension cohomologique des groupes profinis, Topology 3 (1965), 413-420. MR0180619 (31:4853)

[7] J. P. Serre, Une relation dans la cohomologie des p-groupes, C.R. Acad. Sci. Paris 304 (1987), 587-590. MR 897618 (88c:20064)

[8] Y. A. Turygin, A Borsuk-Ulam theorem for $\left(\mathbb{Z}_{p}\right)^{k}$-actions on products of $(\bmod p)$ homology spheres, Topology Appl. 154 (2007), 455-461. MR2278695 (2007j:55005)

[9] E. Yalçın, Group actions and group extensions, Trans. Amer. Math. Soc. 352 (2000), 26892700. MR.1661282 (2000j:57076)

Department of Mathematics, Bilkent University, 06800 Bilkent, Ankara, Turkey

E-mail address: yalcine@fen.bilkent.edu.tr 